\title{
ON CO-MOORE SPACES
}

\author{
MAREK GOLASIŃSKI and DACIBERG LIMA GONÇALVES*
}

\section{Abstract.}

The paper deals with an existence and uniqueness of co-Moore spaces. We show (Theorem 1.2) the uniqueness of a co-Moore space of type $(\mathrm{A}, n)$ for $n \geq 3$ with given homology groups and deduce that finitely generated abelian groups are the only abelian groups for which there exists a co-Moore space.

Then Moore spaces which are also co-Moore are investigated. We present (Proposition 2.1) some necessary conditions on what cohomology groups may appear for spaces those are Moore and co-Moore. At the end, an existence of a co-Moore space of type $(\mathrm{R} / \mathrm{Z}, n+1)$ for $n \geq 2$ is established.

It is well-known ([6]) that for each pair $(\mathrm{A}, n)$, where $\mathrm{A}$ is an abelian group and $n \geq 2$ an integer there is a unique (up to the homotopy type) Moore space $M(\mathrm{~A}, n)$ of type $(\mathrm{A}, n)$. Similarly, a simply connected space $M^{\prime}(\mathrm{A}, n)$ with non-vanishing the reduced integral cohomology group $\mathrm{A}$ in dimension $n$ is called a co-Moore space of type $(\mathrm{A}, n)$. This note concerns with the problem of which type $(\mathrm{A}, n)$ can be realized as a co-Moore space and when they are unique up to the homotopy type. In [8] Kainen, P.C. derives some results on co-Moore spaces of type $(\mathrm{A}, n)$ when $A$ is a finitely generated abelian group and $n \geq 3$. As he pointed out the space $M^{\prime}(\mathrm{A}, n)$ is unique (up to the homotopy type) in this case and a co-Moore space of type $(\mathrm{Q}, n)$ does not exist for any $n \geq 2$, where $\mathrm{Q}$ is the additive group of rationals (cf. also [10]). More generally, in [11] are analysed sequences of groups (with emphasis on the countable case) which can be cohomology groups of a space. Some work on this subject has also been done in [5] provided Godel's Axiom of Constructibility $V=L$ is assumed.

In section 1 we develop (Theorem 1.2) the uniqueness (up to the homotopy type) of a co-Moore space of type $(\mathrm{A}, n)$ for $n \geq 3$ with given homology groups. Then we deduce some its applications and by means of [11], in Corollary 1.5 we show that finitely generated abelian groups are the only coun-

* The first author is indebted to the São Paulo University for its hospitality and to the FAPESPSão Paulo (Brasil) for the financial support during the time that this work was done.

Received April 25, 1996. 
table abelian groups for which there exists a co-Moore space of type $(\mathrm{A}, n)$ for $n \geq 3$.

Section 2 concerns Moore spaces which are co-Moore as well. We make some comments how it is related to the Whitehead problem and examine (Proposition 2.1 and Corollary 2.2) a class of groups A such that $\operatorname{Hom}(A, Z)=0$, where $Z$ is the additive group of integers. In particular, we obtain (without an assumption on Continuum Hypothesis) a description (Proposition 2.1) of divisible abelian groups $A$ of cardinality $\aleph_{1}$ or exp $\aleph_{0}$ for which there is a co-Moore space of type $(\mathrm{A}, n+1)$ for some $n \geq 2$. Finally, we show the existence of a co-Moore of type $(\mathrm{R} / \mathrm{Z}, n+1)$ for any $n \geq 2$, where $\mathbf{R}$ is the additive group of reals which is a Moore space as well.

\section{Existence and uniqueness of some co-Moore spaces.}

First recall ([7, page 109]) that for an abelian group $A$ the conditions $\operatorname{Hom}(A, Z)=0$ and $\operatorname{Ext}(A, Z)=0$ suffice to deduce that $A=0$. Therefore, from the split short exact sequence

$$
0 \rightarrow \operatorname{Ext}\left(H_{k-1} X, \mathbf{Z}\right) \rightarrow H^{k} X \rightarrow \operatorname{Hom}\left(H_{k} X, \mathbf{Z}\right) \rightarrow 0
$$

given by the Universal Coefficient Theorem one derives that a simply connected space $X$ is a co-Moore space of type $(\mathrm{A}, n)$ if $\widetilde{H}_{k} X=0$ for $k \neq n-1, n$ and the abelian groups $\mathrm{A}_{1}=H_{n-1} X$ and $\mathrm{A}_{2}=H_{n} X$ are such that

1) $\mathrm{A}=H^{n} X=\operatorname{Ext}\left(\mathrm{A}_{1}, \mathrm{Z}\right) \oplus \operatorname{Hom}\left(\mathrm{A}_{2}, \mathrm{Z}\right)$,

2) $\operatorname{Hom}\left(A_{1}, Z\right)=0$ and $\operatorname{Ext}\left(A_{2}, Z\right)=0$.

For any abelian group $A$ the group $\operatorname{Hom}(A, Z)$ is contained in a direct product $\prod Z$ of copies of $Z$, namely the group $Z^{A}$ of all maps from $A$ to $Z$ and for a torsion-free group $A$ the group $\operatorname{Ext}(A, Z)$ is divisible. If however $A$ is torsion and $\mathrm{Q}$ is the additive group of the rationals then the exactness of

$$
0 \rightarrow \mathrm{Z} \rightarrow \mathrm{Q} \rightarrow \mathrm{Q} / \mathrm{Z} \rightarrow 0
$$

induces exactness of

$$
0 \rightarrow \operatorname{Hom}(A, Q / Z) \rightarrow \operatorname{Ext}(A, Z) \rightarrow \operatorname{Ext}(A, Q)=0 .
$$

Hence $\operatorname{Ext}(A, Z)=\operatorname{Hom}(A, Q / Z)=\operatorname{Hom}(A, R / Z)$, where $R$ is the additive group of reals. If we give $A$ the discrete topology then $\operatorname{Hom}(A, R / Z)$ is the compact character group of $A$. Thus, if $A$ is finite then $\operatorname{Ext}(A, Z)=A$ and by [9] $\operatorname{card} \operatorname{Ext}(A, Z)=\exp \operatorname{card}(A)$, if $A$ is infinite.

Now let $M(\mathrm{~A}, n)$ be the (unique up to the homotopy type) Moore space of type (A, $n)$ for $n \geq 2$ and $X$ a simply connected space. Then, by the Universal Coefficient Theorem for homotopy groups ([6]), we have the following exact sequence 


$$
0 \rightarrow \operatorname{Ext}\left(\mathrm{A}, \pi_{n+1} X\right) \rightarrow[M(\mathrm{~A}, n), X] \stackrel{\eta}{\rightarrow} \operatorname{Hom}\left(\mathrm{A}, \pi_{n} X\right) \rightarrow 0,
$$

where $\eta$ associates to a homotopy class $[f]$ of a map $f$ the induced homomorphism on the $n$-th homotopy group. Note that we can easily derive the sequence above from the cofibre sequence for some map of bunches of spheres. In particular, if $X$ is the Moore space $M(\mathrm{~B}, n)$ of type $(\mathrm{B}, n)$ then ([1]) $\pi_{n+1} M(\mathrm{~B}, n)=\Gamma(\mathrm{B})$ if $n=2$ and $\pi_{n+1} M(\mathrm{~B}, n)=\mathrm{B} \otimes \mathrm{Z}_{2}$ if $n \geq 3$, where $\Gamma$ is J.H.C. Whitehead's quadratic functor. Thus we get the following short exact sequence

$$
0 \rightarrow \operatorname{Ext}\left(\mathrm{A}, \mathrm{B} \otimes \mathrm{Z}_{2}\right) \rightarrow[M(\mathrm{~A}, n), M(\mathrm{~B}, n)] \stackrel{\eta}{\rightarrow} \operatorname{Hom}(\mathrm{A}, \mathrm{B}) \rightarrow 0
$$

for $n \geq 3$. From the Whitehead Theorem it follows that $\eta$ associates to a homotopy class $[f]$ of a map $f$ the induced homomorphism on the $n$-th homology groups. To state the theorem on the homotopy type of co-Moore spaces we need the following lemma.

Lemma 1.1. If $\mathrm{A}$ is an abelian group such that $\operatorname{Ext}(\mathrm{A}, \mathrm{Z})=0$ then $\operatorname{Ext}\left(A, A^{\prime}\right)=0$ for any $p$-torsion abelian group $\mathrm{A}^{\prime}$, where $p$ is a prime. In particular, $\operatorname{Ext}\left(\mathrm{A}, \mathrm{B} \otimes \mathrm{Z}_{2}\right)=0$ for any abelian group $\mathrm{B}$.

Proof. If $\operatorname{Ext}(A, Z)=0$ then from the cohomology exact sequence induced by the short exact sequence $0 \rightarrow \mathbf{Z} \stackrel{p}{\rightarrow} \mathbf{Z} \rightarrow \mathbf{Z}_{p} \rightarrow 0$ it follows that $\operatorname{Ext}\left(A, Z_{p}\right)=0$ as well. Therefore, $\operatorname{Ext}\left(A, \prod Z_{p}\right)=\prod \operatorname{Ext}\left(A, Z_{p}\right)=0$ for any product $\prod Z_{p}$.

If now $\mathrm{A}^{\prime}$ is a $p$-torsion abelian group then it is a $\mathrm{F}_{p}$-vector space, where $\mathrm{F}_{p}$ is the simple field of characteristic $p$. Therefore, $A^{\prime}=\bigoplus Z_{p}$ over some index set and we get the split short exact sequence of $F_{p}$-vector spaces

$$
0 \rightarrow \mathrm{A}^{\prime} \rightarrow \prod \mathrm{Z}_{p} \rightarrow \mathrm{A}^{\prime \prime} \rightarrow 0,
$$

where the product $\prod Z_{p}$ is indexed by the same set and $A^{\prime \prime}$ is the quotient group. Hence, the group $A^{\prime}$ is a direct summand of $\prod Z_{p}$ and $\operatorname{Ext}\left(A, A^{\prime}\right)=0$.

We now can show the main theorem.

TheOREm 1.2. A simply connected space $X$ is a co-Moore space of type $(\mathrm{A}, n)$, for $n \geq 3$ if and only if it is homotopy equivalent to the wedge $M\left(\mathrm{~A}_{1}, n-1\right) \vee M\left(\mathrm{~A}_{2}, n\right)$, where $\mathrm{A}=\operatorname{Ext}\left(\mathrm{A}_{1}, \mathrm{Z}\right) \oplus \operatorname{Hom}\left(\mathrm{A}_{2}, \mathrm{Z}\right), \operatorname{Hom}\left(\mathrm{A}_{1}, \mathrm{Z}\right)=$ 0 and $\operatorname{Ext}\left(A_{2}, Z\right)=0$ for some abelian groups $A_{1}$ and $A_{2}$.

Poof. $\Rightarrow$ : Let $X$ be a co-Moore space of type $(\mathrm{A}, n)$ and $\mathrm{A}_{1}=H_{n-1} X$, $\mathrm{A}_{2}=H_{n} X$. Then $\operatorname{Hom}\left(\mathrm{A}_{1}, \mathrm{Z}\right)=0, \operatorname{Ext}\left(\mathrm{A}_{2}, \mathrm{Z}\right)=0$ and $\mathrm{A}=\operatorname{Ext}\left(\mathrm{A}_{1}, \mathrm{Z}\right) \oplus$ $\operatorname{Hom}\left(\mathrm{A}_{2}, \mathrm{Z}\right)$. From the homology decomposition ([6]) it follows that $X$ has the homotopy type of the mapping cone $M\left(\mathrm{~A}_{1}, n-1\right) \cup_{\tau} C M\left(\mathrm{~A}_{2}, n-1\right)$ of 
some map $\tau: M\left(\mathrm{~A}_{2}, n-1\right) \rightarrow M\left(\mathrm{~A}_{1}, n-1\right)$. But $n-1 \geq 2$, hence from the long homology exact sequence determined by the map $\tau$ we get that the induced homomorphism $\tau_{*}: \mathrm{A}_{2} \rightarrow \mathrm{A}_{1}$ on the $(n-1)$-th homology group is trivial. So, by the Universal Coefficient Theorem for homotopy groups and Lemma 1.1 it follows that the homotopy class $[\tau]$ is an element of $\operatorname{Ext}\left(\mathrm{A}_{2}, \mathrm{~A}_{1} \otimes \mathrm{Z}_{2}\right)=0$. Hence the map $\tau$ is homotopy trivial and the space $M\left(\mathrm{~A}_{1}, n-1\right) \cup_{\tau} C M\left(\mathrm{~A}_{2}, n-1\right)$ has the homotopy type of the wedge $M\left(\mathrm{~A}_{1}, n-1\right) \vee M\left(\mathrm{~A}_{2}, n\right)$.

$\Leftarrow$ : Let a space $X$ has the homotopy type of the wedge $M\left(\mathrm{~A}_{1}, n-1\right) \vee M\left(\mathrm{~A}_{2}, n\right)$. Then $H_{n-1} X=\mathrm{A}_{1}, H_{n} X=\mathrm{A}_{2}$ and $H_{k} X=0$ for $k \neq n-1, n$. Thus, by the Universal Coefficient Theorem $H^{n} X=\operatorname{Ext}\left(\mathrm{A}_{1}, \mathrm{Z}\right) \oplus \operatorname{Hom}\left(\mathrm{A}_{2}, \mathrm{Z}\right)$, where $\operatorname{Hom}\left(\mathrm{A}_{1}, \mathrm{Z}\right)=0, \operatorname{Ext}\left(\mathrm{A}_{2}, \mathrm{Z}\right)=0$ and $H^{k} X=0$ for $k \neq n$.

Let now $\mathrm{A}$ be a finitely generated abelian group. Then $\mathrm{A}=f(\mathrm{~A}) \oplus t(\mathrm{~A})$, where $f(\mathrm{~A})$ and $t(\mathrm{~A})$ denote its free and torsion part, respectively. But, $\operatorname{Ext}(f(\mathrm{~A}), \mathbf{Z})=0$ and $\operatorname{Hom}(t(\mathrm{~A}), \mathbf{Z})=0$. Therefore, by Theorem 1.2 the space $X=M(t(\mathrm{~A}), n-1) \vee M(f(\mathrm{~A}), n)$ is the unique (up to the homotopy type) coMoore space of type $(\mathrm{A}, n)$ such that $H_{n-1} X=t(\mathrm{~A})$ and $H_{n} X=f(\mathrm{~A})$ for $n \geq 2$. Conversely, we can show the following proposition.

Proposition 1.3. For $n \geq 3$, if A finitely generated then $X$ is a co-Moore space of type $(\mathrm{A}, n)$ if and only if it is homotopy equivalent to the wedge $M(t(\mathrm{~A}), n-1) \vee M(f(\mathrm{~A}), n)$.

Proof. Let $\mathrm{A}_{1}=H_{n-1} X$ and $\mathrm{A}_{2}=H_{n} X$. Then by Theorem 1.2 the space $X$ has the homotopy type of the wedge $M\left(\mathrm{~A}_{1}, n-1\right) \vee M\left(\mathrm{~A}_{2}, n\right)$ for $n \geq 3$. Thus, $H^{n} X=\mathrm{A}=f(\mathrm{~A}) \oplus t(\mathrm{~A})=\operatorname{Ext}\left(\mathrm{A}_{1}, \mathrm{Z}\right) \oplus \operatorname{Hom}\left(\mathrm{A}_{2}, \mathrm{Z}\right)$, where $\operatorname{Hom}\left(A_{1}, Z\right)=0$ and $\operatorname{Ext}\left(A_{2}, Z\right)=0$.

We show that $\mathrm{A}_{1}=t(\mathrm{~A})$ and $\mathrm{A}_{2}=f(\mathrm{~A})$. The group $\operatorname{Ext}\left(\mathrm{A}_{1}, \mathrm{Z}\right)$ is finitely generated (as a subgroup of $A$ ) and let $t\left(A_{1}\right)$ denote the torsion part of $A_{1}$. Then, the short exact sequence

$$
0 \rightarrow t\left(\mathrm{~A}_{1}\right) \rightarrow \mathrm{A}_{1} \rightarrow \mathrm{A}_{1} / t\left(\mathrm{~A}_{1}\right) \rightarrow 0
$$

gives rise to the exact cohomology sequence

$$
0 \rightarrow \operatorname{Ext}\left(A_{1} / t\left(A_{1}\right), Z\right) \rightarrow \operatorname{Ext}\left(A_{1}, Z\right) \rightarrow \operatorname{Ext}\left(t\left(A_{1}\right), Z\right) \rightarrow 0 .
$$

The group $A_{1} / t\left(A_{1}\right)$ is torsion-free, so the group $\operatorname{Ext}\left(A_{1} / t\left(A_{1}\right), Z\right)$ is divisible and it is a direct summand of the finitely generated group $\operatorname{Ext}\left(A_{1}, Z\right)$. Therefore $\operatorname{Ext}\left(\mathrm{A}_{1} / t\left(\mathrm{~A}_{1}\right), \mathrm{Z}\right)=0$. But $\operatorname{Hom}\left(\mathrm{A}_{1} / t\left(\mathrm{~A}_{1}\right), \mathrm{Z}\right)=\operatorname{Hom}\left(\mathrm{A}_{1}, \mathrm{Z}\right)=0$, so $A_{1} / t\left(A_{1}\right)=0$ and $A_{1}=t\left(A_{1}\right)$. By [9], we have $\operatorname{card} \operatorname{Ext}\left(A_{1}, Z\right)=$ expcard $\left(A_{1}\right)$ if $A_{1}$ is an infinite torsion group. Hence $A_{1}$ is a finite torsion 
group and $\operatorname{Ext}\left(A_{1}, Z\right)=A_{1}$. On the other hand, the group $\operatorname{Hom}\left(A_{2}, Z\right)$ is finitely generated and torsion-free, so it is free. But

$$
\mathrm{A}=f(\mathrm{~A}) \oplus t(\mathrm{~A})=\operatorname{Ext}\left(\mathrm{A}_{1}, \mathrm{Z}\right) \oplus \operatorname{Hom}\left(\mathrm{A}_{2}, \mathrm{Z}\right)=\mathrm{A}_{1} \oplus \operatorname{Hom}\left(\mathrm{A}_{2}, \mathrm{Z}\right),
$$

hence $\mathrm{A}_{1}=t(\mathrm{~A})$ and $\operatorname{Hom}\left(\mathrm{A}_{2}, \mathrm{Z}\right)=f(\mathrm{~A})$. Moreover, any free generator of the group $f(\mathrm{~A})$ determines a non-trivial splitting map $\mathrm{A}_{2} \rightarrow Z$ (i.e. its image is a direct summand of $\left.A_{2}\right)$. So, $A_{2}=A_{2}^{\prime} \oplus f(A)$ for some abelian group $A_{2}^{\prime}$ and $\operatorname{Hom}\left(\mathrm{A}_{2}, \mathrm{Z}\right)=\operatorname{Hom}\left(\mathrm{A}_{2}^{\prime}, \mathrm{Z}\right) \oplus f(\mathrm{~A})=f(\mathrm{~A})$. Thus $\operatorname{Hom}\left(\mathrm{A}_{2}^{\prime}, \mathrm{Z}\right)=0$ and $\operatorname{Ext}\left(\mathrm{A}_{2}, \mathrm{Z}\right)=\operatorname{Ext}\left(\mathrm{A}_{2}^{\prime} \oplus f(\mathrm{~A}), \mathrm{Z}\right)=\operatorname{Ext}\left(\mathrm{A}_{2}^{\prime}, \mathrm{Z}\right)=0$. Finally $\mathrm{A}_{2}^{\prime}=0$, by [7, page 109], and $\mathrm{A}_{2}=f(\mathrm{~A})$.

This result has been stated in [8] but the proof presented above follows from our tools. Unfortunately, for a given abelian group $\mathrm{A}$ and $n \geq 2$ there does not exist in general a co-Moore space of type $(\mathrm{A}, n)$. In particular, if $\mathrm{F}$ is a free abelian group of rank greater or equal $\aleph_{1}$ we do not know if there exists a co-Moore space of type (F,n) for any $n \geq 2$. In [11] the following theorem is stated.

THEOREM 1.4. If $X$ is a space such that the cohomology groups $H^{n} X$ and $H^{n+1} X$ are countable then the homology group $H_{n} X$ is finitely generated.

In particular, we can deduce the result.

COROLLARY 1.5. If $\mathrm{A}$ is a countable group then there is a co-Moore space of type $(\mathrm{A}, n)$ for $n \geq 2$ if and only if $\mathrm{A}$ is finitely generated.

Proof. If the group $A$ is finitely generated then by Proposition 1.3 there exists a unique (up to the homotopy type) a co-Moore space of type $(\mathrm{A}, n)$ for $n \geq 2$.

Let now A be a countable group and $X$ a co-Moore space of type $(\mathrm{A}, n)$ for some $n \geq 2$. Then, by Theorem 1.4 the homology groups $H_{n-1} X$ and $H_{n} X$ are finitely generated. Hence, by the Universal Coefficient Theorem the group $H^{n} X=\mathrm{A}$ is finitely generated as well.

\section{Moore spaces which are co-Moore.}

Let $M(\mathrm{~A}, n)$ be a Moore space of type $(\mathrm{A}, n)$. Then $H^{n} M(\mathrm{~A}, n)=\operatorname{Hom}(\mathrm{A}, \mathrm{Z})$, $H^{n+1} M(\mathrm{~A}, n)=\operatorname{Ext}(\mathrm{A}, \mathrm{Z})$ and $H^{k} M(\mathrm{~A}, n)=0$ for $k \neq n, n+1$. Therefore, the space $M(\mathrm{~A}, n)$ is a co-Moore space if and only if:

1) $\operatorname{Ext}(A, Z)=0$ or

2) $\operatorname{Hom}(A, Z)=0$.

If $\operatorname{Ext}(\mathrm{A}, \mathrm{Z})=0$ then the group $\mathrm{A}$ is called a $W$-group and the Whitehead problem asks whether every $\mathrm{W}$-group is free. The Stein-Serre theorem $([3,7,14])$ says that the answer is "yes" for groups of countable rank. In a 
remarkable paper S. Shelah ([13]) proved that the Whitehead problem is unsolvable in ordinary set theory. More precisely, he shows that the different answers to the problem are obtained depending on which of some two additional axioms are added to set theory.

We now analyse the class of groups $A$ such that $\operatorname{Hom}(A, Z)=0$ and remind the reader of some results. The first reasonable subclass $T_{0}$ of these groups is given by the property that any element of a group $\mathrm{A}$ in $T_{0}$ has infinitely many integral divisors. In particular, any divisible or torsion group and any (non-free) subgroup of the rationals is in the class $T_{0}$. If $\mathrm{A}$ is a torsion group then from section 1 it follows that $\operatorname{Ext}(A, Z)=\operatorname{Hom}(A, R / Z)$ and the Moore space $M(\mathrm{~A}, n)$ is a co-Moore space of type $(\operatorname{Hom}(\mathrm{A}, \mathrm{R} / \mathrm{Z}), n+1)$ for $n \geq 2$. The group $\operatorname{Hom}(A, R / Z)$ is finite if $A$ is finite and $\operatorname{card} \operatorname{Hom}(A, R / Z)=\exp \operatorname{card}(A)$, if $A$ is infinite. By [4, Chapter IV] any divisible group is isomorphic to the direct sum of copies of $Q$ and of copies of the Prüfer group $Z_{p^{\infty}}$ for various primes $p$. The $\operatorname{group} \operatorname{Ext}(\mathbf{Q}, \mathbf{Z})$ is isomorphic ([7, page 109]) to the additive group of the reals $R$ and the group $\mathbf{Z}_{p^{\infty}}$ is the direct limit of the monomorphisms $\mathbf{Z}_{p^{n}} \rightarrow \mathbf{Z}_{p^{n+1}}$ induced by $\mathbf{Z} \stackrel{p}{\rightarrow} \mathbf{Z}$. Then, $\lim _{\leftarrow} \operatorname{Ext}\left(\mathbf{Z}_{p^{n}}, \mathbf{Z}\right)=\lim \mathbf{Z}_{p^{n}}=\mathbf{Z}_{p}^{\wedge}$, where $\mathbf{Z}_{p}^{\wedge}$ is the additive group of the $p$ adic integers. Hence by [12], there is a natural short exact sequence

$$
0 \rightarrow \lim _{\leftarrow}{ }^{1} \operatorname{Hom}\left(\mathbf{Z}_{p^{n}}, \mathbf{Z}\right) \rightarrow \operatorname{Ext}\left(\mathbf{Z}_{p^{\infty}}, \mathbf{Z}\right) \rightarrow \mathbf{Z}_{p}^{\wedge} \rightarrow 0
$$

and thus $\operatorname{Ext}\left(Z_{p^{\infty}}, \mathbf{Z}\right)=\mathbf{Z}_{p}^{\wedge}$. Therefore, for any divisible group $A$ the group $\operatorname{Ext}(A, Z)$ is isomorphic to the direct product of copies of $\mathrm{R}$ and $\mathrm{Z}_{p}^{\wedge}$ for various $p$ and the Moore space $M(\mathrm{~A}, n)$ is a co-Moore space of type $(\operatorname{Ext}(\mathrm{A}, \mathrm{Z}), n+1)$ for $n \geq 2$.

Let now $A$ be any non-free subgroup of $Q$. Then, the group $\operatorname{Ext}(A, Z)$ is divisible, of cardinality $\exp \aleph_{0}\left(\left[7\right.\right.$, page 105]) and $\mathrm{A} / p \mathrm{~A}=0$ or $\mathrm{A} / p \mathrm{~A}=\mathrm{Z}_{p}$ for any prime $p$, where $Z_{p}$ is the cyclic group of order $p$. If $A / p A=Z_{p}$ then exactness of

$$
0 \rightarrow \mathrm{A} \stackrel{p}{\rightarrow} \mathrm{A} \rightarrow \mathrm{Z}_{p} \rightarrow 0
$$

induces exactness of

$$
0 \rightarrow \operatorname{Ext}\left(Z_{p}, Z\right)=Z_{p} \rightarrow \operatorname{Ext}(A, Z) \stackrel{p}{\rightarrow} \operatorname{Ext}(A, Z) \rightarrow 0 .
$$

Hence the only one copy of the Prüfer group $Z_{p^{\infty}}$ appears as a direct summand of the divisible group $\operatorname{Ext}(\mathrm{A}, \mathrm{Z})$. Let $P$ denote the set of all primes $p$ such that the group $A$ is not $p$-divisible. Then,

$$
\operatorname{Ext}(\mathrm{A}, \mathrm{Z})=\bigoplus_{2^{\aleph_{0}}} \mathrm{Q} \oplus \bigoplus_{p \in P} \mathrm{Z}_{p^{\infty}}=\mathrm{R} \oplus \bigoplus_{p \in P} \mathrm{Z}_{p^{\infty}} .
$$


We now present some necessary conditions on what cohomology groups may appear for spaces those are Moore and co-Moore.

Proposition 2.1. Let $\mathrm{A}$ a torsion-free abelian group such that $\operatorname{Hom}(\mathrm{A}, \mathrm{Z})$ $=0$.

1) If $\mathrm{A}$ is not $p$-divisible for some prime $p$ then either a finite or uncountable number of copies of the Prüfer group $\mathrm{Z}_{p^{\infty}}$ appears in the decomposition of the divisible group $\operatorname{Ext}(\mathrm{A}, \mathrm{Z})$.

2) If $\operatorname{card} \operatorname{Ext}(\mathrm{A}, \mathrm{Z}))=\aleph_{1}$ or $\left.\operatorname{card} \operatorname{Ext}(\mathrm{A}, \mathrm{Z})\right)=\exp \aleph_{0}$ (e.g. $\mathrm{A}$ is of finite rank which is not free or $\mathrm{A}$ is $p$-divisible for some prime $p$ ) then only an uncountable number of copies of the group of rationals $\mathrm{Q}$ appears in the decomposition of the divisible group $\operatorname{Ext}(\mathrm{A}, \mathrm{Z})$.

Proof. 1) If $A$ is not $p$-divisible then $A / p A$ is a direct sum of copies of $Z_{p}$. Exactness of

$$
0 \rightarrow \mathrm{A} p \rightarrow \mathrm{A} \rightarrow \mathrm{A} / p \mathrm{~A} \rightarrow 0
$$

induces exactness of

$$
0 \rightarrow \operatorname{Ext}(\mathrm{A} / p \mathrm{~A}, \mathrm{Z}) \rightarrow \operatorname{Ext}(\mathrm{A}, \mathrm{Z}) \stackrel{p}{\rightarrow} \operatorname{Ext}(\mathrm{A}, \mathrm{Z}) \rightarrow 0 .
$$

But $\operatorname{Ext}(\mathrm{A} / p \mathrm{~A}, \mathrm{Z})$ is isomorphic to the direct product of a number of copies of $Z_{p}$ and the kernel of the map $\operatorname{Ext}(A, Z) \stackrel{p}{\rightarrow} \operatorname{Ext}(A, Z)$ is isomorphic to the direct sum of copies of $Z_{p}$. Hence either a finite or uncountable number of copies of the Prifer group $Z_{p \infty}$ could appears in the decomposition of the group $\operatorname{Ext}(A, Z)$.

2) Suppose that only a countable number of $Q$ appears in the decomposition of $\operatorname{Ext}(A, Z)$ and $A$ is not $\aleph_{1}$-free. Then, there exists a non-free subgroup $A^{\prime}$ (of countable rank) of $A$. By Pontrjagin's Lemma ([4, page 51]) there exists a non-free subgroup $A^{\prime \prime}$ (of finite rank) of $A^{\prime}$. If $A^{\prime \prime}$ is of rank 1 then, by the above result, an uncountable number of $\mathrm{Q}$ appears in the decomposition of $\operatorname{Ext}\left(A^{\prime \prime}, Z\right)$. Proceeding inductively on rank of $A^{\prime \prime}$ we get the same for $\operatorname{Ext}\left(A^{\prime \prime}, Z\right)$, where $A^{\prime \prime}$ is of finite rank. Thus an uncountable number of $\mathrm{Q}$ appears in the decomposition of $\operatorname{Ext}(A, Z)$ as well and we obtain a contradiction. Therefore, the group $A$ is $\aleph_{1}$-free and card $A \geq \aleph_{1}$ (since the group $\operatorname{Ext}(A, Z)$ is non-trivial).

Now by Zorn's Lemma, select a maximal pure independent subset $X$ of $A$ and let $F$ be the subgroup of $A$ generated by $X$, which is then free and pure in A. The group $A$ is $\aleph_{1}$-free, hence cardF $\geq \aleph_{0}$ and suppose that card $F=\aleph_{0}$. The exact sequence $0 \rightarrow \mathrm{F} \rightarrow \mathrm{A} \rightarrow \mathrm{A} / \mathrm{F} \rightarrow 0$ gives rise to the exact cohomology sequence 


$$
0 \rightarrow \operatorname{Hom}(F, Z) \rightarrow \operatorname{Ext}(A / F, Z) \rightarrow \operatorname{Ext}(A, Z) \rightarrow 0 .
$$

Thus it follows that $\operatorname{card} \operatorname{Ext}(A / F, Z)=\exp \aleph_{0}$.

Let $v(\mathrm{~A} / \mathrm{F})$ denote the intersection of all subgroups of $\mathrm{A} / \mathrm{F}$ such that the corresponding quotients are $\aleph_{1}$-free. Then, since by [2, Theorem 1.8] $\operatorname{card} \operatorname{Ext}(\mathrm{A} / \mathrm{F}, \mathrm{Z})=\exp \aleph_{0} \geq \exp [\operatorname{card} v(\mathrm{~A} / \mathrm{F})]$, it follows that $\operatorname{card} v(\mathrm{~A} / \mathrm{F}) \leq$ $\aleph_{0}$. Put $\bar{F}$ for the subgroup of $A$ defined by the condition $\bar{F} / F=v(A / F)$ then from the proof of Theorem 3.3 in [2] one gets that $\bar{F}=A$. From the exact sequence $0 \rightarrow \mathrm{F} \rightarrow \mathrm{A} \rightarrow v(\mathrm{~A} / \mathrm{F}) \rightarrow 0$ it follows that card $\mathrm{F}=$ card $\mathrm{A} \geq \aleph_{1}$ contradicting that card $\mathrm{F}=\aleph_{0}$. Therefore, card $\mathrm{F} \geq \aleph_{1}$. If $p$ is a prime then $p \mathrm{~F}=\mathrm{F} \cap p \mathrm{~A}$, by purity of $\mathrm{F}$. Hence

$$
\mathrm{F} / p \mathrm{~F}=\mathrm{F} / \mathrm{F} \cap p \mathrm{~A}=\mathrm{F}+p \mathrm{~A} / p \mathrm{~A} \subseteq \mathrm{A} / p \mathrm{~A} .
$$

Since card $\mathrm{F} \geq \aleph_{1}$, we have that $\operatorname{dim}_{F_{p}} \mathrm{~A} / p \mathrm{~A} \geq \aleph_{1}$, where $\mathrm{F}_{p}$ is the simple field of characteristic $p$. The exact sequence $0 \rightarrow \mathrm{A} \stackrel{p}{\rightarrow} \mathrm{A} \rightarrow \mathrm{A} / p \mathrm{~A} \rightarrow 0$ gives rise to the exact cohomology sequence

$$
0 \rightarrow \operatorname{Ext}(\mathrm{A} / p \mathrm{~A}, \mathrm{Z}) \rightarrow \operatorname{Ext}(\mathrm{A}, \mathrm{Z}) \stackrel{p}{\rightarrow} \operatorname{Ext}(\mathrm{A}, \mathrm{Z}) \rightarrow 0 .
$$

Finally, the contradiction $\aleph_{1}=\operatorname{card} \operatorname{Ext}(\mathrm{A}, \mathrm{Z}) \geq \operatorname{card} \operatorname{Ext}(\mathrm{A} / p \mathrm{~A}, \mathrm{Z}) \geq \exp \aleph_{1}$ ends the proof.

Let now $\mathrm{A}$ be any abelian group. Then exactness of $0 \rightarrow t(\mathrm{~A}) \rightarrow \mathrm{A} \rightarrow$ $\mathrm{A} / t(\mathrm{~A}) \rightarrow 0$ induces exactness of the split sequence

$$
0 \rightarrow \operatorname{Ext}(\mathrm{A} / t(\mathrm{~A}), \mathrm{Z}) \rightarrow \operatorname{Ext}(\mathrm{A}, \mathrm{Z}) \rightarrow \operatorname{Ext}(t(\mathrm{~A}), \mathrm{Z}) \rightarrow 0 .
$$

But $\operatorname{Ext}(t(\mathbf{A}), \mathbf{Z})=\operatorname{Hom}(t(\mathbf{A}), \mathbf{R} / \mathbf{Z})$, where $\mathbf{R}$ is the additive group of reals. Thus, $\operatorname{Ext}(t(\mathrm{~A}), \mathbf{Z})=t(\mathrm{~A})$, if $t(\mathrm{~A})$ is finite and by [9] we get $\operatorname{card} \operatorname{Ext}(t(\mathrm{~A}), \mathbf{Z})$ $=\exp \operatorname{card} t(\mathrm{~A})$, if $t(\mathrm{~A})$ is infinite. Then we can state the following corollary.

Corollary 2.2. Let $\mathrm{A}$ be an abelian group such that $\operatorname{Hom}(\mathrm{A}, \mathrm{Z})=0$ and $\operatorname{card} \operatorname{Ext}(\mathrm{A}, \mathrm{Z})=\aleph_{1} \quad\left(\right.$ resp. $\left.\quad \operatorname{card} \operatorname{Ext}(\mathrm{A}, \mathrm{Z})=\exp \aleph_{0}\right)$. Then $\operatorname{Ext}(\mathrm{A}, \mathrm{Z})=$ $\operatorname{Ext}(\mathrm{A} / t(\mathrm{~A}), \mathrm{Z}) \oplus \operatorname{Ext}(t(\mathrm{~A}), \mathrm{Z}) \quad$ with $\operatorname{card} t(\mathrm{~A})<\aleph_{0} \quad\left(\right.$ resp. $\left.\quad \operatorname{card} t(\mathrm{~A})=\aleph_{0}\right)$, where $\operatorname{Ext}(\mathrm{A} / t(\mathrm{~A}), \mathrm{Z})$ is a divisible group with properties given by Proposition 2.1 .

Let now $t_{1}(\mathrm{~A})$ be the subgroup of $\mathrm{A}$ generated by all its elements with infinitely many divisors. If $t_{1}(\mathrm{~A})=\mathrm{A}$ then it is easy to see that $\operatorname{Hom}(\mathrm{A}, \mathrm{Z})=0$ and we put $T_{1}$ for the class of all such groups. Of course, $T_{0} \subsetneq T_{1}$ since for instance, the groups $\prod Z_{p}$ and $\prod Z_{p}^{\wedge}$ given by the products of cyclic groups and the additive groups of the $p$-adic integers, respectively for all primes $p$ 
are in the class $T_{1}$ but not in $T_{0}$. This leads to a more general construction. For a group A consider the following transfinite sequence of its subgroups

$$
t_{1}(\mathrm{~A}) \subseteq t_{2}(\mathrm{~A}) \subseteq \cdots \subseteq t_{\alpha}(\mathrm{A}) \subseteq \cdots \subseteq \mathrm{A},
$$

where $t_{1}\left(\mathrm{~A} / t_{\alpha}(\mathrm{A})\right)=t_{\alpha+1}(\mathrm{~A}) / t_{\alpha}(\mathrm{A})$ and $t_{\alpha}(\mathrm{A})=\bigcup_{\xi<\alpha} t_{\xi}(\mathrm{A})$ if $\alpha$ is a limit ordinal.

Let $T_{\alpha}$ be the class of all groups $\mathrm{A}$ such that $t_{\alpha}(\mathrm{A})=\mathrm{A}$. Then we get a transfinite sequence of the following classes of groups

$$
T_{0} \subseteq T_{1} \subseteq T_{2} \subseteq \cdots \subseteq T_{\alpha} \subseteq \cdots .
$$

By induction on $n$ we show that $T_{n} \subsetneq T_{n+1}$ for all $n \geq 1$. Let $\mathrm{A}_{1}=\prod \mathrm{Z}_{p}$, where $p$ runs over all primes and the element $e_{1} \in A_{1}$ is given by the sequence of generators of all groups $\mathbf{Z}_{p}$. Then the group $\mathbf{A}_{2}(p)=\mathbf{A}_{1} \oplus \mathbf{Z} /\left(e_{1},-p\right) \mathbf{Z}$ for any prime $p$ is in the class $T_{2}$ but not in $T_{1}$.

Given groups $\mathrm{A}_{n}(p)=\mathrm{A}_{n-1} \oplus \mathbf{Z} /\left(e_{n-1},-p\right) \mathbf{Z}$, for all primes $p$ which are in the class $T_{n}$ but not in $T_{n-1}$ consider the product $\mathrm{A}_{n}=\prod \mathrm{A}_{n}(p)$, where $p$ runs over all primes $p$. Take the element $e_{n} \in \mathrm{A}_{n}$ given by the sequence of elements $(0,1)+\left(e_{n-1},-p\right) \mathbf{Z}$ for all primes $p$, where $(0,1) \in \mathbf{A}_{n-1} \oplus \mathbf{Z}$. Then the group $\mathrm{A}_{n+1}(p)=\mathrm{A}_{n} \oplus \mathbf{Z} /\left(e_{n},-p\right) \mathbf{Z}$ for any prime $p$ is in the class $T_{n+1}$ but not in $T_{n}$.

Lemma 2.3. If $X$ is a co-Moore space of type $(\mathrm{A}, n)$, for $n \geq 2$ then $A=\operatorname{Ext}\left(A_{1}, Z\right) \oplus \operatorname{Hom}\left(A_{2}, Z\right)$ for some abelian groups $A_{1}$ and $A_{2}$, where the group $\operatorname{Ext}\left(\mathrm{A}_{1}, \mathrm{Z}\right)$ has generators with infinitely many integral divisors. Furthermore, this decomposition of the group $\mathrm{A}$ is unique up to isomorphism.

Proof. Exactness of

$$
0 \rightarrow \mathrm{Z} \rightarrow \mathrm{Q} \rightarrow \mathrm{Q} / \mathrm{Z} \rightarrow 0
$$

induces exactness of

$$
0 \rightarrow \operatorname{Hom}\left(A_{1}, Q\right) \rightarrow \operatorname{Hom}\left(A_{1}, Q / Z\right) \rightarrow \operatorname{Ext}\left(A_{1}, Z\right) \rightarrow 0 .
$$

But $\mathrm{Q} / \mathbf{Z}=\bigoplus \mathbf{Z}_{p^{\infty}}$, where $p$ runs over all primes and $\mathbf{Z}_{p^{\infty}}$ is the Prüfer group of type $p^{\infty}$. Hence

$$
\operatorname{Hom}\left(A_{1}, \mathbf{Q} / \mathbf{Z}\right)=\operatorname{Hom}\left(A_{1}, Z_{2^{\infty}}\right) \oplus \operatorname{Hom}\left(A_{1}, \bigoplus_{p \neq 2} Z_{p^{\infty}}\right)
$$

The group $Z_{2 \infty}$ is $p$-divisible for any prime $p \neq 2$ and $\bigoplus_{p \neq 2} Z_{p^{\infty}}$ is $2^{n}$-divisible for any natural $n \geq 1$. Thus, the group $\operatorname{Ext}\left(\mathrm{A}_{1}, \mathrm{Z}\right)$ has generators with infinitely many divisors as a quotient of the group $\operatorname{Hom}\left(A_{1}, Q / Z\right)$.

For suppose that $A=B_{1} \oplus B_{2}=B_{1}^{\prime} \oplus B_{2}^{\prime}$, where the groups $B_{1}, B_{1}^{\prime}$ have 
generators with infinitely many divisors and $B_{2}, B_{2}^{\prime}$ are contained in a direct product $\prod Z$. Then $B_{1}=B_{1}^{\prime}$ and $B_{2}=A / B_{1}=A / B_{2}^{\prime}=B_{2}^{\prime}$.

We now can state the next result.

Proposition 2.4. 1) If $\mathrm{A}$ is an abelian group and $t_{\alpha}(\mathrm{A})=\mathrm{A}$ for some ordinal $\alpha$ then $\operatorname{Hom}(\mathrm{A}, \mathrm{Z})=0$.

2) If $X$ is a co-Moore space of type $(\mathrm{A}, n)$ and $t_{\alpha}(\mathrm{A})=\mathrm{A}$ for some ordinal $\alpha$ then $\alpha=1$.

Proof. 1) Note that $\operatorname{Hom}(\mathrm{A}, \mathrm{Z})=\operatorname{Hom}\left(\mathrm{A} / t_{\alpha}(\mathrm{A}), \mathrm{Z}\right)$ for any ordinal $\alpha$.

2) From section 1 it follows that there are abelian groups $A_{1}$ and $A_{2}$ such that $A=\operatorname{Ext}\left(A_{1}, Z\right) \oplus \operatorname{Hom}\left(A_{2}, Z\right)$. Thus, by Lemma 2.3 we get $t_{1}(A)=$ $\operatorname{Ext}\left(\mathrm{A}_{1}, \mathrm{Z}\right)$ and $t_{1}\left(\mathrm{~A} / t_{1}(\mathrm{~A})\right)=t_{2}(\mathrm{~A}) / t_{1}(\mathrm{~A})=t_{1}\left(\operatorname{Hom}\left(\mathrm{A}_{2}, \mathrm{Z}\right)\right)=0$. Finally, $t_{2}(\mathrm{~A})=t_{1}(\mathrm{~A})$ and $\mathrm{A}=t_{\alpha}(\mathrm{A})=t_{1}(\mathrm{~A})$.

Unfortunately, the classes $T_{\alpha}$ do not exhaust all groups $A$ such that $\operatorname{Hom}(A, Z)=0$. Fuchs and Loonstra ([4, page 128]) constructed a torsionfree group $A$ of rank 2 with the following properties:

1) every subgroup of rank 1 is cyclic;

2) every torsion-free quotient of rank 1 is divisible.

First, note that $t_{1}(\mathrm{~A})=0$ for this group. For let $t_{1}(\mathrm{~A}) \neq 0$ and take $a \in t_{1}(\mathrm{~A}), a \neq 0$. Consider the subgroup $\mathrm{A}_{a}$ of $\mathrm{A}$ generated by all elements $a^{\prime} \in \mathrm{A}$ such that $a=n a^{\prime}$ for some $n \in \mathrm{Z}$. Then rank of $\mathrm{A}_{a}$ is 1 , so the subgroup $\mathrm{A}_{a}$ is cyclic and we have a contradiction since the group $Z$ does not contain non-zero elements with infinitely many divisors.

If $\operatorname{Hom}(A, Z) \neq 0$ then $A=A^{\prime} \oplus Z$ for some subgroup $A^{\prime}$ of $A$. Thus $A^{\prime}$ is of rank 1 and is not divisible. Therefore, the Moore space $M(A, n)$ is a coMoore space of type $(\operatorname{Ext}(\mathrm{A}, \mathrm{Z}), n+1)$. To find $\operatorname{Ext}(\mathrm{A}, \mathrm{Z})$ note that there is a short exact sequence

$$
0 \rightarrow \mathrm{Z} \rightarrow \mathrm{A} \rightarrow \mathrm{Q} \rightarrow 0
$$

where $\mathrm{Q}$ is the additive group of the rationals which derives the following one

$$
0 \rightarrow \mathrm{Z} \rightarrow \operatorname{Ext}(\mathrm{Q}, \mathrm{Z}) \rightarrow \operatorname{Ext}(\mathrm{A}, \mathrm{Z}) \rightarrow 0 .
$$

But the group $\operatorname{Ext}(\mathrm{O}, \mathrm{Z})$ is isomorphic ([7, page 109]) to the additive group of the reals $R$ hence, $\operatorname{Ext}(A, Z)=R / Z$. Thus, we may state

Proposition 2.5. For any $n \geq 2$, there exists a co-Moore space of type $(\mathrm{R} / \mathrm{Z}, n+1)$, where $\mathrm{R}$ is the additive group of reals and $\mathrm{Z}$ the group of integers.

Finally, we close with the following problem. 
Problem 2.6. Describe all divisible abelian groups $A$ for which there is a co-Moore space of type $(\mathrm{A}, n)$ for some $n \geq 2$.

\section{REFERENCES}

1. H. J. Baues, Quadratic functors and metastable homotopy, J. Pure Appl. Alg. 91 (1994), 49107.

2. S. Chase, On group extensions and a problem of J.H.C. Whitehead, Proceedings of the Symposium on Abelian Groups Topics in abelian groups, 173-193, edited by Irwin, J. M., Walker, E. A., Scott, Foresman and Company (1963).

3. A. Ehrenfeucht, On a problem of J.H.C. Whitehead concerning abelian groups, Bull. Acad. Polon. Sci. 3 (1955), 127-128.

4. L. Fuchs, Infinite Abelian Groups, New York-London; Academic Press (1973).

5. H. L. Hiller, M. Huber and S. Shelah, The structure of $\operatorname{Ext}(\mathrm{A}, \mathrm{Z})$ and $V=L$, Math. Z. 162 (1978), 39-50.

6. P. J. Hilton, Homotopy theory and duality, New York-London-Paris; Science Publishers (1965).

7. P. J. Hilton and U. Stammbach, A Course in Homological Algebra, New York-HeidelbergBerlin; Springer-Verlag (1971).

8. P. C. Kainen, Every commutative square of abelian groups can be realized by a homotopy commutative square of Moore, or co-Moore spaces, J. London Math. Soc. 2 (1972), 577583.

9. S. Kakutani, On cardinal numbers related with a compact abelian group, Proc. Imp. Acad. Tokyo 19 (1943), 366-372.

10. D. M. Kan and G. W. Whitehead, On the realizibility of singular cohomology groups, Proc. Amer. Math. Soc. 12 (1961), 24-25.

11. R. J. Nunke and J. J. Rotman, Singular cohomology groups, J. London Math. Soc. 37 (1962), 301-306.

12. J. E. Roos., Sur les foncteurs dérivés de lim, C. R. Acad. Sci. Paris 252 (1961), 3702-3704.

13. S. Shelah, Infinite abelian groups, Whitehead problem and some constructions, Israel J. Math. 18 (1974), 243-256.

14. K. Stein, Analytische Funktionen mehrerer komplexer Veränderlichen zu vorgegebenen Periodizitätsmoduln und das zweite Cousinsche Problem, Math. Ann. 123 (1951), 201-222.

FACULTY OF MATHEMATICS AND INFORMATICS NICHOLAS COPERNICUS UNIVERSITY

CHOPINA $12 / 18,87-100$ TORUŃ

POLAND

e-mail: marek@mat.uni.torun.pl
DEPARTMENT OF MATHEMATICS-IME

UNIVERSITY OF SAO PAULO

CAIXA POSTAL 66.281-AG. CIDADE DE SAO PAULO 05315-970 SAO PAULO,

BRASIL

e-mail: dlgoncal@ime.usp.br 